\title{
EGFR L747P mutation in one lung adenocarcinoma patient responded to afatinib treatment: a case report
}

\author{
Tong Zhou ${ }^{1}$, Xiaoyue Zhou $^{1}$, Peng Li $^{1}$, Chuang Qi $^{2}$, Yang Ling ${ }^{1}$ \\ ${ }^{1}$ Department of Medical Oncology, Changzhou Cancer Hospital of Soochow University, Changzhou 213032, China; ${ }^{2}$ The Medical Department, 3D \\ Medicines Inc., Shanghai 201100, China \\ Correspondence to: Yang Ling. Department of Medical Oncology, Changzhou Cancer Hospital of Soochow University, No. 68 Honghe Road, Xinbei \\ District, Changzhou 213032, China. Email: lingy_cz@126.com.
}

Submitted Oct 26, 2018. Accepted for publication Dec 05, 2018.

doi: $10.21037 /$ jtd.2018.12.26

View this article at: http://dx.doi.org/10.21037/jtd.2018.12.26

\section{Introduction}

EGFR mutation is an important driving event in the development of non-small cell lung cancer (NSCLC), especially in pathologically diagnosed lung adenocarcinoma and non-smoking women in Asia (1). Compared with traditional platinum-containing chemotherapy, NSCLC patients with certain EGFR sensitive mutations showed higher clinical response to EGFR tyrosine kinase inhibitors (EGFR-TKIs), as confirmed by a series of phase III clinical trials, such as WJTOG3405, OPTIMAL, LUXLUNG6 and so on.

The frequency of EGFR mutations is about $50 \%$ in Asian lung adenocarcinoma patients, which is higher than in the European populations (1). The sensitive mutations of EGFR gene mainly concentrate in exon 18-21. Mutations in these regions change spatial structure of the enzyme functional domain, and lead to signal activation (2). According to relevant studies, exon 21 point mutation and exon 19 deletion account for $85 \%$ of the whole EGFR alterations $(1,2)$. However, gene sequencing revealed some rare EGFR mutations existing. Except that EGFR Gly719Xaa, Leu861Gln, and Ser768Ile were proved to be sensitive to second-generation EGFR-TKI drug, understanding of other rare mutations still remain enigma (3). More studies should be initiated to explore whether some of them have effects on clinical medication selection. Here, we reported one lung adenocarcinoma patient with EGFR L747P mutation had achieved a remarkable progression-free survival (PFS) after afatinib treatment.

\section{Case presentation}

One 44-year-old female patient was admitted to hospital for accidental head injury in March 2015. However, a soft tissue density mass was observed in the right pulmonary apical segment and the right parietal lobe of brain was occupied after chest CT and cranial MRI examination, respectively (Figure 1A,B). Meanwhile, the patient was accompanied with polycystic liver and kidney. Emission computerized tomography (ECT) examination revealed normal skeletal metabolism imaging with no sigh of bone metastasis. Pulmonary biopsy was applied to apex of right lung with a pathological diagnosis of lung adenocarcinoma (Figure 1C). According to results of these comprehensive examinations, advanced NSCLC with brain metastasis was diagnosed. Afterwards, common driver genes were tested for seeking targeted drugs by amplification refractory mutation system (AMRS) or real-time PCR (RT-PCR) methods, while the status of EGFR, ALK, ROS1 and RET were negative.

Chemotherapy with four cycles of pemetrexed combined with carboplatin were administered for first-line standard treatment, followed by one cycle of pemetrexed for standard maintenance chemotherapy and $\gamma$-knife treatment for brain lesions. The patient came to hospital in January 2016 as one regular follow-up visit and chest CT revealed increase of right upper lung lesion. Disease progression was considered.

To seek potential targeted drugs, next-generation sequencing (NGS) analysis was performed in February 2016, and revealed one rare EGFR mutation (L747P), which was located in exon 19 (Figure 2A). Mutation 

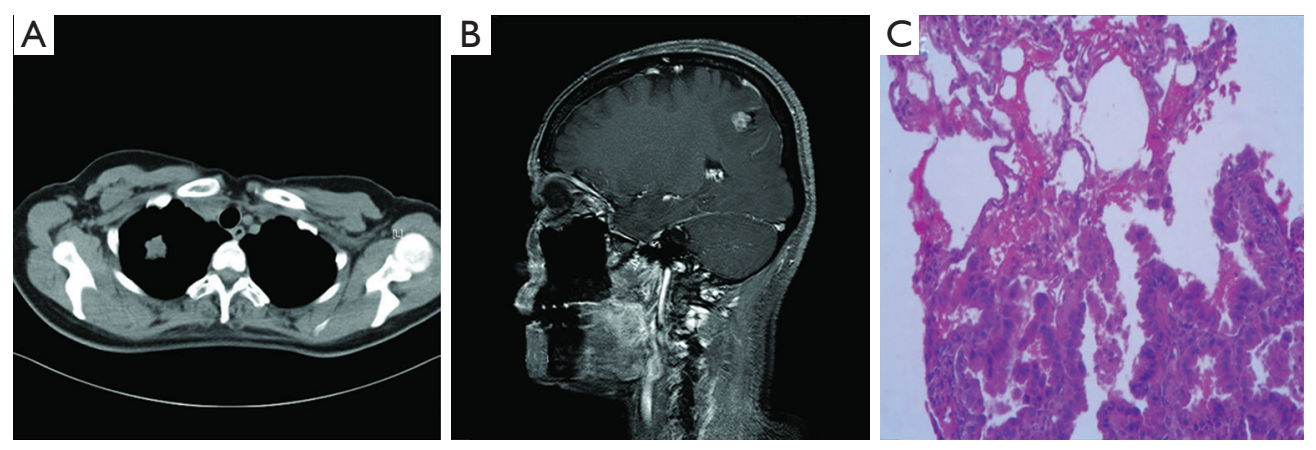

Figure 1 Chest CT, histopathological, and cranial MRI findings of the primary lung (A and B) and metastatic brain (C) tumor. (A) Chest CT revealed a soft tissue density mass existed in the right pulmonary apical segment; (B) cranial MRI examination indicated the right parietal lobe of brain was occupied; (C) pulmonary biopsy was applied to apex of right lung with a pathological diagnosis of lung adenocarcinoma (hematoxylin-eosin staining: 400x).

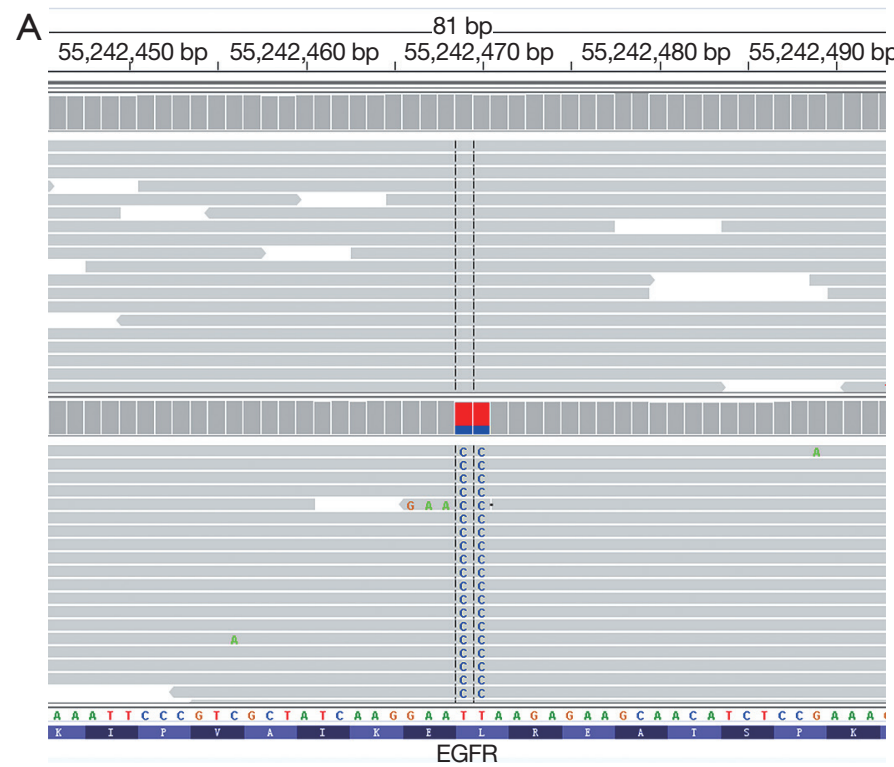

\begin{tabular}{lcl}
\hline Bene & Mutation site & Mutation style \\
\hline ADH1B & H48R & Heterozygous polymorphism \\
ALDH2 & E457K & Heterozygous polymorphism \\
CDA & K27Q & Heterozygous polymorphism \\
CYP2D6 & P34S & Heterozygous polymorphism \\
CYP3A5 & CYP3A5*3 & Heterozygous polymorphism \\
DPYD & I543V & Heterozygous polymorphism \\
EGFR & L747P & Point mutation (mutation frequency: 29\%) \\
& R521K & Homozygous polymorphism \\
ERCC1 & N118N & Homozygous polymorphism \\
GSTT1 & - & Homozygous deletion polymorphism \\
MSH6 & Y850X & Germinal truncation mutation \\
MTHFR & A222V & Heterozygous polymorphism \\
NQO1 & P187S & Heterozygous polymorphism \\
RB1 & c.G265-1T & Point mutation (mutation frequency: 21\%) \\
TP53 & V216M & Point mutation (mutation frequency: 21\%) \\
& P72R & Heterozygous polymorphism \\
TYMS & $-6 \mathrm{bp} /-6 \mathrm{bp}$ & Homozygous deletion polymorphism \\
XRCC1 & Q399R & Heterozygous polymorphism \\
\hline & &
\end{tabular}

Figure 2 Next-generation sequencing (NGS) findings of the primary lung tumor tissue samples (A and B). (A) EGFR L747P point mutation located in terminal hydrophobic center of $\beta$ chain of $\mathrm{N}$ terminal in kinase domain. (B) Heterozygotic polymorphism was found in genes of ADH1B H48R, ALDH2 E457K, CDA K27Q, CYP2D6 P34S, CYP3A5 CYP3A5*3, DPYD I543V, MTHFR A222V, NQO1 P187S, TP53 P72R, and XRCC1 Q399R. Homozygous polymorphism was found in genes of EGFR R521K and ERCC1N118N. Homozygous deletion polymorphism was found in genes of GSTT1 and TYMS -6bp/-6bp. Other gene mutations were found in MSH6 Y850X phylogenetic truncation mutation, and point mutation of RB1 c.G265-1T and TP53 V216M. 

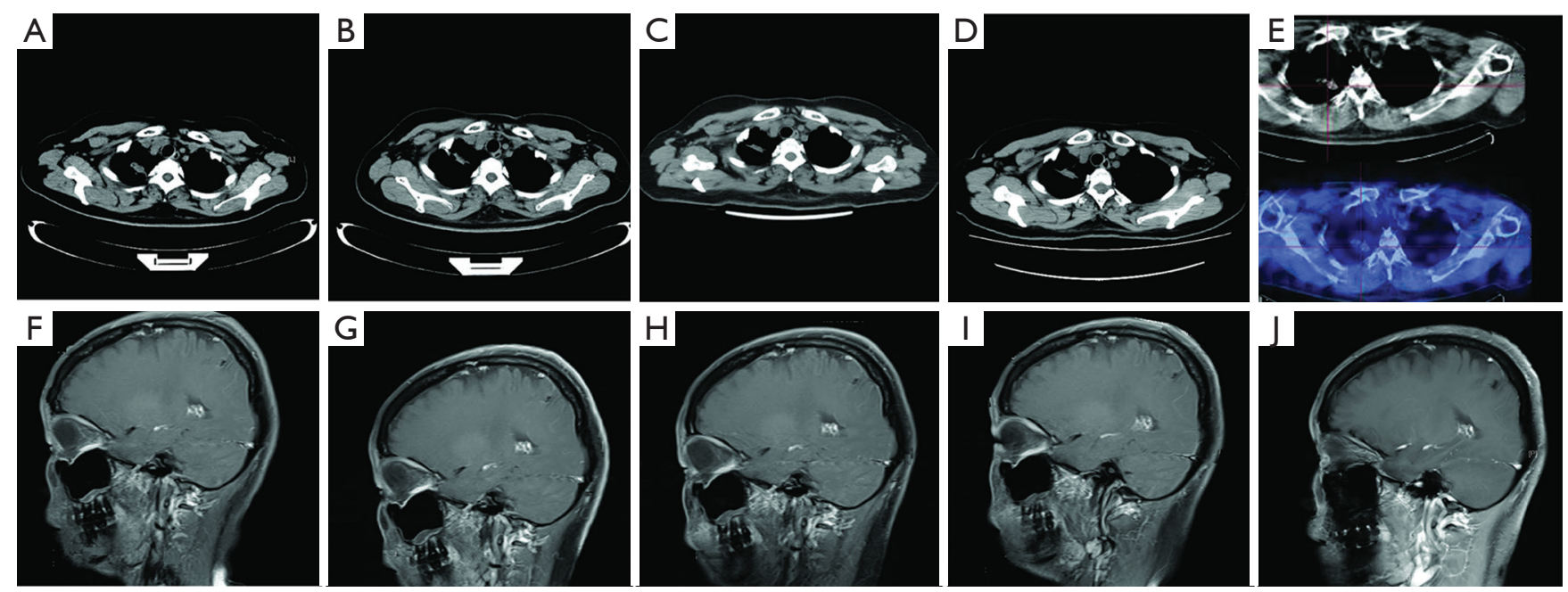

Figure 3 The dynamic evolution of the patient's primary lung tumor (A,B,C,D,E) and brain metastasis (F,G,H,I,J) during afatinib treatment. (A) The baseline chest CT scan (January, 2016) showing the lesion of right upper lung before afatinib treatment; (B,C,D) the lesions in right upper lobe showed a SD in several follow-up visits by chest CT scan; (E) SPET examination indicated the lesion of right upper lung still kept stable without metabolic activity (March 2018); (F) the baseline MRI scan (February, 2016) showing the right parietal lobe of brain was occupied before afatinib treatment; $(\mathrm{G}, \mathrm{H}, \mathrm{I})$ the lesions in right parietal lobe of brain showed a SD in several follow-up visits during afatinib treatment; (J) MRI examination indicated the lesions in right parietal lobe of brain were still stable (April 2018).

profiling of this patient was summarized in Figure $2 B$. Beginning from March 2016, oral afatinib was administered $(40 \mathrm{mg} / \mathrm{d}$ was performed in the first six month, then adjusted to use $30 \mathrm{mg} / \mathrm{d}$ from September 2016 to February 2017, and $20 \mathrm{mg} / \mathrm{d}$ was taken from March 2017 to now). After five months of treatment, the lesion in right upper lobe showed a SD according to the Response Evaluation Criteria in Solid Tumors, version 1.1 (RECIST 1.1) and so was brain metastases status in right parietal lobe (Figure 3). Several follow-up visits were conducted, and the last visit was in April 2018, which showed tumor lesion in the right lung still kept stable and the PFS already exceeded 24 months.

\section{Discussion}

Bioinformatics analysis proved that the EGFR L747P mutation located in terminal hydrophobic center of the $\beta$ chain of the $\mathrm{N}$ terminal in the kinase domain, and that this mutation changed the structure of the hydrophobic center, causing continuous activation of EGFR kinase and potentially contributing to the development of lung cancer.

Three case reports focusing on the relationship between EGFR L747P mutation and clinical efficiency of EGFRTKIs were found in PubMed (4-6). Two cases among them described that lung adenocarcinoma patients with EGFR L747P mutation were not sensitive to the first and third generation EGFR-TKIs $(4,5)$.

To our interest, the author tried to apply afatinib to one lung adenocarcinoma patient with EGFR L747P mutation in the third case (6), but discontinued due to intolerable diarrhea and mucosal ulceration after two weeks afatinib administration. The efficacy of afatinib could not be evaluated in this report. Compared with first-generation EGFR-TKIs, the most common side effects during afatinib therapy were diarrhea, rash and acne, which could be controlled by drug interruption or dosage reduction (7). Based on analysis of the LUX-Lung 3 and LUX-Lung 6, researchers found $\mathrm{mPFS}$ in patients with dosage reduction in the first 6 months after afatinib treatment was similar to the population without a dose-reduction (7). In our case, the afatinib dosage experienced two adjustments because of facial skin rash, and side effects were managed with keeping on oral afatinib therapy up to now.

One post-hoc analysis of LUX-Lung 2, LUX-Lung 3 , and LUX-Lung 6 indicated that lung adenocarcinoma patients harboring uncommon EGFR mutations (Gly719Xaa, Leu861Gln, and Ser768Ile) could benefit from afatinib treatment, with a median PFS ranging from 8.2 to 14.7 months (3). In our case, the patient was still alive with 
a PFS of exceeding 24 months, which indicated the clinical benefits of EGFR L747P mutation might be comparable to EGFR Gly719Xaa, Leu861Gln and Ser768Ile after afatinib therapy.

Beside EGFR L747P mutation, another EGFR R5 $21 \mathrm{~K}$ homozygous polymorphism was identified after NGS analysis. EGFR R521 locates in exon 13, encoding extracellular binding domain of EGFR tyrosine kinase. Generally speaking, only mutations locate in the intracellular domain can activate signaling pathways, and so far none of researches proved that EGFR R521K was sensitive to EGFR-TKIs. Based on current available clinical evidence, other gene mutations of the patient also could not cause a response to EGFR-TKIs.

In conclusion, we presented the first case of one adenocarcinoma patient with EGFR L747P mutation achieved an impressive PFS after afatinib treatment. This discovery might help lung adenocarcinoma patients with EGFR L747P to obtain EGFR-TKIs therapeutic opportunities, but further trials are needed to confirm this finding.

\section{Acknowledgements}

Funding: This study was supported by the Changzhou Sci \&Tech Program, China (Grant No. CE20175037) and the High-level Health Talents of Changzhou City (Grant No. 2016CZLJ009).

\section{Footnote}

Conflicts of Interest: The authors have no conflicts of interest to declare.

Informed Consent: Written informed consent was obtained from the patient for publication of this manuscript and any accompanying images.

\section{References}

1. Shi $\mathrm{Y}, \mathrm{Au} \mathrm{JS}$, Thongprasert S, et al. A prospective, molecular epidemiology study of EGFR mutations in Asian patients with advanced non-small-cell lung cancer of adenocarcinoma histology (PIONEER). J Thorac Oncol 2014;9:154-62.

2. Pao W, Chmielecki J. Rational, biologically based treatment of EGFR-mutant non-small-cell lung cancer. Nat Rev Cancer 2010;10:760-74.

3. Yang JC, Sequist LV, Geater SL, et al. Clinical activity of afatinib in patients with advanced non-small-cell lung cancer harbouring uncommon EGFR mutations: a combined post-hoc analysis of LUX-Lung 2, LUX-Lung 3, and LUX-Lung 6. Lancet Oncol 2015;16:830-8.

4. Wang YT, Ning WW, Li J, et al. Exon 19 L747P mutation presented as a primary resistance to EGFR-TKI: a case report. J Thorac Dis 2016;8:E542-6.

5. Yu G, Xie X, Sun D, et al. EGFR mutation L747P led to gefitinib resistance and accelerated liver metastases in a Chinese patient with lung adenocarcinoma. Int J Clin Exp Pathol 2015;8:8603-6.

6. Huang J, Wang Y, Zhai Y, et al. Non-small cell lung cancer harboring a rare EGFR L747P mutation showing intrinsic resistance to both gefitinib and osimertinib (AZD9291): A case report. Thorac Cancer 2018;9:745-9.

7. Yang JC, Sequist LV, Zhou C, et al. Effect of dose adjustment on the safety and efficacy of afatinib for EGFR mutation-positive lung adenocarcinoma: post hoc analyses of the randomized LUX-Lung 3 and 6 trials. Ann Oncol 2016;27:2103-10.
Cite this article as: Zhou T, Zhou X, Li P, Qi C, Ling Y. EGFR L747P mutation in one lung adenocarcinoma patient responded to afatinib treatment: a case report. J Thorac Dis 2018;10(12):E802-E805. doi: 10.21037/jtd.2018.12.26 